Pacific

Journal of

Mathematics

FUNDAMENTAL SOLUTIONS OF INVARIANT DIFFERENTIAL OPERATORS ON A SEMISIMPLE LIE GROUP II

Guillermo Ames 


\title{
FUNDAMENTAL SOLUTIONS OF INVARIANT DIFFERENTIAL OPERATORS ON A SEMISIMPLE LIE GROUP II
}

\author{
Guillermo Ames
}

\begin{abstract}
Let $G$ be a linear connected semisimple Lie group. We denote by $\mathcal{U}(\mathfrak{g})^{K}$ the algebra of left invariant differential operators on $G$ that are also right invariant by $K$, and $\mathcal{Z}\left(\mathcal{U}(\mathfrak{g})^{K}\right)$ denotes center of $\mathcal{U}(\mathfrak{g})^{K}$.

In this paper we give a sufficient condition for a differential operator $P \in \mathcal{Z}\left(\mathcal{U}(\mathfrak{g})^{K}\right)$ to have a fundamental solution on $G$. This result extends the same one obtained previously for real rank one Lie groups and groups with only one conjugacy class of Cartan subgroups.
\end{abstract}

\section{Introduction.}

Let $G$ be a linear connected semisimple Lie group. The algebra of left invariant differential operators on $G$ is canonically identified with the universal algebra $\mathcal{U}(\mathfrak{g})$. The operators of the center $\mathcal{Z}(\mathfrak{g})$ are the bi-invariant differential operators on $G$. More generally, we consider the algebra $\mathcal{U}(\mathfrak{g})^{K}$ of right $K$-invariant differential operators in $\mathcal{U}(\mathfrak{g})$, where $K$ is a maximal compact subgroup of $G \cdot \mathcal{Z}\left(\mathcal{U}(\mathfrak{g})^{K}\right)$ will denote its center.

We denote by $\mathcal{D}(G)$ the space of $C^{\infty}$ functions with compact support. The dual $\mathcal{D}^{\prime}(G)$ of continuous linear functionals in $\mathcal{D}(G)$ is the space of distributions of $G$.

An operator $P$ in $\mathcal{U}(\mathfrak{g})$ acts on $\mathcal{D}^{\prime}(G)$ in the following way:

$$
P E(f)=E\left(P^{t} f\right),
$$

where $P^{t} \in \mathcal{U}(\mathfrak{g})$ is such that, if $d x$ is a Haar measure on $G$,

$$
\int_{G} P f(x) g(x) d x=\int_{G} f(x) P^{t} g(x) d x .
$$

In addition, if $X \in \mathfrak{g}, X^{t}=-X$, so the $P \mapsto P^{t}$ is the anti-automorphism of $\mathcal{U}(\mathfrak{g})$ extending $-I d$ of $\mathfrak{g}$. Also, this map preserves the subalgebras $\mathcal{Z}(\mathfrak{g})$ and $\mathcal{U}(\mathfrak{g})^{K}$.

Definition 1. A distribution $E \in \mathcal{D}^{\prime}(G)$ is a fundamental solution of a differential operator $P \in \mathcal{U}(\mathfrak{g})$ if $P E=\delta$, where $\delta(f)=f(1)$; and $E$ is a parametrix of $P$ if $P E-\delta \in C^{\infty}(G)$. 
In this paper we extend the main result of [1] for a connected semisimple Lie group with a simply connected complexification. We start by recalling this result as it was stated in [1].

Remember that in general $\mathcal{Z}\left(\mathcal{U}(\mathfrak{g})^{K}\right) \simeq \mathcal{Z}(\mathfrak{g}) \otimes \mathcal{Z}(\mathfrak{k})$ (Knop's Theorem $[\mathbf{9}]$ ). Given $\mathfrak{h}_{0}$ and $\mathfrak{t}_{0}$ Cartan subalgebras of $\mathfrak{g}_{0}$ and $\mathfrak{k}_{0}$ respectively, we will denote $\gamma_{\mathfrak{h}}^{G}$ and $\gamma_{\mathfrak{t}}^{K}$ the Harish-Chandra homomorphisms of $\mathcal{Z}(\mathfrak{g})$ and $\mathcal{Z}(\mathfrak{k})$ with respect to the subalgebras $\mathfrak{h}$ and $\mathfrak{t}$ : Then we have

$$
\begin{aligned}
& \mathcal{Z}(\mathfrak{g}) \times \mathcal{Z}(\mathfrak{k}) \stackrel{\gamma_{\mathfrak{h}}^{G} \times \gamma_{\mathfrak{t}}^{K}}{\longrightarrow} \mathcal{U}(\mathfrak{h})^{W} \times \mathcal{U}(\mathfrak{t})^{W_{K}} \\
& \downarrow \otimes \quad \downarrow \otimes \\
& \mathcal{Z}\left(\mathcal{U}(\mathfrak{g})^{K}\right) \stackrel{\simeq}{\longrightarrow} \mathcal{Z}(\mathfrak{g}) \otimes \mathcal{Z}(\mathfrak{k}) \stackrel{\gamma_{\mathfrak{h}}^{G} \otimes \gamma_{\mathfrak{t}}^{K}}{\longrightarrow} \mathcal{U}(\mathfrak{h})^{W} \otimes \mathcal{U}(\mathfrak{t})^{W_{K}} \stackrel{i \otimes i}{\longrightarrow} \mathcal{U}(\mathfrak{h} \oplus \mathfrak{t}) .
\end{aligned}
$$

Therefore, by the way of the homomorphisms described above, we can associate to $P \in \mathcal{Z}\left(\mathcal{U}(\mathfrak{g})^{K}\right)$ a differential operator $\left(\gamma_{\mathfrak{h}}^{G} \otimes \gamma_{\mathfrak{t}}^{K}\right)(P)$ in the group $H \times T$, where $H$ and $T$ are the respective Cartan subgroups of $G$ and $K$ with Lie algebras $\mathfrak{h}_{0}$ and $\mathfrak{t}_{0}$.

We say that a Cartan subgroup $H$ of $G$ is fundamental if it contains a $G$-conjugate of a Cartan subgroup of $K$. All fundamental Cartan subgroups of $G$ are conjugate.

We will denote $\gamma^{G}=\gamma_{\mathfrak{h}}^{G}$, where $\mathfrak{h}_{0}$ is the Lie subalgebra of a fundamental Cartan subgroup $H$. Because in $K$ all Cartan subgroups are conjugate, we put $\gamma^{K}=\gamma_{t}^{K}$.

We can now state our main result:

Theorem 1.1. Let $G$ be a connected semisimple Lie group with a simply connected complexification. Let $H$ be a fundamental Cartan subgroup of $G$ and $P \in \mathcal{Z}\left(\mathcal{U}(\mathfrak{g})^{K}\right)$. If $\left(\gamma^{G} \otimes \gamma^{K}\right)(P)$ has a fundamental solution in $H \times T$, then $P$ has a fundamental solution in $G$.

When $P$ is a bi-invariant operator, we obtain a complete proof of the Theorem announced in [2] (see [3] for another proof of this result):

Corollary 1.2 (Benabdallah-Rouvière). Let $P \in \mathcal{Z}(\mathfrak{g})$. If $\gamma^{G}(P)$ has a fundamental solution in $H$, then $P$ has a fundamental solution in $G$.

The proof of Theorem 1.1 goes by explicit construction of the fundamental solution of $P$, using the Plancherel formula as the main tool.

\section{Preliminaries.}

In this section we fix notation and summarize some known facts about representation theory that will be needed through this paper. We refer to $[\mathbf{1}]$ for any unexplained notation.

2.1. Notation. Let $G$ be a linear connected semisimple Lie group, $\mathfrak{g}_{0}$ its Lie algebra. The complexification of any real Lie algebra will be denoted without the subscript. 
We will assume that $G$ has a simply connected complexification, that is, $G$ is the analytic subgroup corresponding to $\mathfrak{g}_{0}$ of the simply connected complex group with Lie algebra $\mathfrak{g}$.

$\theta$ will denote a Cartan involution in either $\mathfrak{g}_{0}, \mathfrak{g}$ or $G$. Let $\mathfrak{g}_{0}=\mathfrak{k}_{0} \oplus \mathfrak{p}_{0}$ be a Cartan decomposition of $\mathfrak{g}_{0}$ with respect to $\theta$; that is, $\mathfrak{k}_{0}=\left\{X \in \mathfrak{g}_{0}\right.$ : $\theta X=X\}$ and $\mathfrak{p}_{0}=\left\{X \in \mathfrak{g}_{0}: \theta X=-X\right\}$.

If $K$ is the analytic subgroup of $G$ with Lie algebra $\mathfrak{k}_{0}, K$ is a maximally compact subgroup of $G$. We fix $\mathfrak{t}_{0}$ a Cartan subalgebra of $\mathfrak{k}_{0}$ coming from a maximal torus $T$ of $K$.

On $\mathfrak{g}$ we define the inner product, $(X, Y)=-B(X, J \theta Y)$, where $B$ is the Killing form of $\mathfrak{g}$ and $J$ is conjugation with respect to $\mathfrak{g}_{0}$.

Let $H$ be any $\theta$-stable Cartan subgroup. Then $\mathfrak{h}_{0}=\mathfrak{b}_{0}^{H} \oplus \mathfrak{a}_{0}^{H}$, with $\mathfrak{b}_{0}^{H} \subseteq \mathfrak{k}_{0}, \mathfrak{a}_{0}^{H} \subseteq \mathfrak{p}_{0}$. We can associate to $H$ a cuspidal parabolic subgroup $Q^{H}$ with Langlands decomposition $M^{H} A^{H} N^{H}$. $B^{H}$.

The group $M^{H}$ is a reductive Lie group with compact Cartan subgroup

To avoid overloading the notation, we will not use superscripts for these subgroups when working with a fixed $H$ or whenever is clear from the context.

2.2. Discrete series of $M$ (cf. $\left[8\right.$, XII.8]). Let $M_{0}$ be the identity component of $M, Z_{M}$ the center of $M$, and define $M^{\#}=M_{0} Z_{M}$.

If $\alpha \in \Delta\left(\mathfrak{g}_{0}, \mathfrak{h}_{0}\right)$ is a real root, let $\gamma_{\alpha}=\exp 2 \pi i H_{\alpha}$, where $H_{\alpha} \in \mathfrak{a}$ is the standard co-root. If $F(B)$ is the group generated by all the $\gamma_{\alpha}$, then $F(B)$ is a finite abelian subgroup of $Z_{M}$. It also holds $M^{\#}=M_{0} F(B)$.

Discrete series representation of $M^{\#}$ are parametrized by pairs $(\lambda, \chi)$ such that:

1) $\lambda$ is a discrete series parameter of $M_{0}$; that is, $\lambda \in i \mathfrak{b}_{0}^{\prime}$ is nonsingular and $\lambda-\rho_{M}$ is analytically integral, or equivalently, $\lambda$ is analytically integral, because $\rho_{M}$ is.

2) $\chi \in F(B)$.

3) $\chi=e^{\lambda-\rho_{M}}$ on $B \cap F(B)$.

We denote $\pi^{\#}(\lambda, \chi)$ the respective discrete series representation of $M^{\#}$, which has the following properties:

1) $\pi^{\#}(\lambda, \chi)$ has infinitesimal character $\lambda$.

2) $\pi^{\#}(\lambda, \chi) \simeq \pi^{\#}\left(\lambda^{\prime}, \chi^{\prime}\right)$ if and only if $\chi=\chi^{\prime}$ and $\lambda^{\prime}=w \lambda$ for some $w \in W\left(B_{0}, M_{0}\right)$.

Discrete series representations of $M$ are exactly the representations

$$
\pi(\lambda, \chi)=\operatorname{Ind}_{M \#}^{M} \pi^{\#}(\lambda, \chi) .
$$

Properties 1 and 2 above also hold for $\pi(\lambda, \chi)$.

As in the connected case, $\lambda$ is called the Harish-Chandra parameter of $\pi(\lambda, \chi)$ (or $\left.\pi^{\#}(\lambda, \chi)\right)$. We will denote $\mathcal{S}_{d}(M)$ the set of all pairs $(\lambda, \chi)$ that 
defines a discrete series representation of $M$ and $\mathcal{S}_{d}\left(M_{0}\right)$ the set of HarishChandra parameters $\lambda$.

2.3. $H$-series of $G$. Given $\pi(\lambda, \chi)$ a discrete series representation of $M$, $\nu \in \mathfrak{a}^{\prime}$ a complex linear functional on $\mathfrak{a}$, the $H$-series of $G$ consists of the representations

$$
\begin{aligned}
\pi(H, \lambda, \chi, \nu) & =\operatorname{Ind}_{M A N}^{G}\left(\pi(\lambda, \chi) \otimes e^{\nu} \otimes 1\right) \\
& =\operatorname{Ind}_{M A A N}^{G}\left(\pi^{\#}(\lambda, \chi) \otimes e^{\nu} \otimes 1\right) .
\end{aligned}
$$

$\pi(H, \lambda, \chi, \nu)$ has infinitesimal character $\lambda+\nu$ relative to $\mathfrak{h}_{0}$. We will denote its global character by $\Theta(H, \lambda, \chi, \nu)$. The unitary $H$-series is the subset when $\nu$ takes pure imaginary values on $\mathfrak{a}_{0}$.

2.4. Plancherel formula. We can now write down the Plancherel formula for semisimple groups. Let $\operatorname{Car}(G)$ denote a set of representatives of $\theta$-stables Cartan subgroups.

Theorem 2.1. There is a nonnegative function $m(H, \lambda, \chi, \nu)$ defined in $\mathcal{S}_{d}(M) \times i \mathfrak{a}_{0}^{\prime}$ such that

$$
\delta=\sum_{H \in \operatorname{Car}(G)}\left(\sum_{(\lambda, \chi) \in \mathcal{S}_{d}(M)} \int_{\nu \in i \mathfrak{a}_{0}^{\prime}} \Theta(H, \lambda, \chi, \nu) m(H, \lambda, \chi, \nu) d \nu\right) .
$$

The function $m(H, \lambda, \chi, \nu)$ has the following properties:

(i) For each $(\lambda, \chi) \in \mathcal{S}_{d}(M), m(H, \lambda, \chi, \nu)$ is the restriction to $i \mathfrak{a}_{0}^{\prime}$ of a meromorphic function on $\mathfrak{a}^{\prime}$ without poles on $i \mathfrak{a}_{0}^{\prime}$.

(ii) Exist a positive constant $C$ and a positive integer $l$ such that for all $(\lambda, \chi) \in \mathcal{S}_{d}(M), \nu \in i \mathfrak{a}_{0}^{\prime}$, we have

$$
|m(H, \lambda, \chi, \nu)| \leq C\left(1+|\lambda|^{2}\right)^{l}\left(1+|\nu|^{2}\right)^{l} .
$$

We refer to [5, Theorem 6.17] for a more general and explicit statement of the above formula, and to [6, Lemma 3.3] for the inequality (ii).

\section{Action of $P$ on characters.}

If $\pi$ is an admissible representation with global character $\Theta_{\pi}$ and infinitesimal character $\chi_{\pi}$, and $P \in \mathcal{Z}(\mathfrak{g})$ is a bi-invariant differential operator, then $P \Theta_{\pi}=\chi_{\pi}(P) \Theta_{\pi}([8$, Prop. 10.24]).

In [1] we prove a similar result for an operator $P \in \mathcal{Z}\left(\mathcal{U}(\mathfrak{g})^{K}\right)$. We recall it here. If $E \in \mathcal{D}^{\prime}(G)$ and $\tau \in \hat{K}$ is an irreducible unitary representation of $K$, then $E^{\tau}$ denotes its isotypic component.

Proposition 3.1. If $\pi$ is an admissible representation with infinitesimal character $\chi_{\pi}$ and global character $\Theta_{\pi}$, and $P$ is a differential operator in $\mathcal{Z}\left(\mathcal{U}(\mathfrak{g})^{K}\right)$, then

$$
P \Theta_{\pi}^{\tau}=\left(\chi_{\pi} \otimes \chi_{\tau}\right)(P) \Theta_{\pi}^{\tau} \text {. }
$$




\section{Inversion of infinitesimal characters.}

Let $H \in \operatorname{Car}(G), P \in \mathcal{Z}\left(\mathcal{U}(\mathfrak{g})^{K}\right), \lambda \in \mathfrak{b}^{\prime}, \mu \in \mathfrak{t}^{\prime}$. We will set

$$
P(H, \lambda, \mu, \nu)=\left(\chi_{\lambda+\nu} \otimes \chi_{\mu}\right)(P)=\left(\left(\gamma_{\mathfrak{h}}^{G} \otimes \gamma^{K}\right)(P)\right)(\lambda+\nu, \mu) .
$$

So if $\pi(H, \lambda, \chi, \nu)$ is an $H$-series representation, $\tau \in \hat{K}$ with infinitesimal character $\mu_{\tau} \in i \mathfrak{t}_{0}^{\prime}$, and $P \in \mathcal{Z}\left(\mathcal{U}(\mathfrak{g})^{K}\right)$, we denote

$$
P(H, \lambda, \tau, \nu)=P\left(H, \lambda, \mu_{\tau}, \nu\right)=\left(\left(\gamma_{\mathfrak{h}}^{G} \otimes \gamma^{K}\right)(P)\right)\left(\lambda+\nu, \mu_{\tau}\right) .
$$

Notice that this equation is independent of $\chi$. With this notation, Proposition 3.1 can be written

$$
P \Theta(H, \lambda, \chi, \nu)=P(H, \lambda, \tau, \nu) \Theta^{\tau}(H, \lambda, \chi, \nu) .
$$

Observe that $P(H, \lambda, \tau, \nu)$ is a polynomial function of $\nu \in \mathfrak{a}^{\prime}$, and we will denote it $P(H, \lambda, \tau)$. Also, if $P \in \mathcal{Z}(\mathfrak{g})$ or in $\mathcal{Z}(\mathfrak{k})$, the expression $P(H, \lambda, \tau, \nu)$ simplifies to $P(H, \lambda, \nu)$ or $P(\tau)$, respectively.

Proposition 4.1. Given $P \in \mathcal{Z}\left(\mathcal{U}(\mathfrak{g})^{K}\right)$ and $H \in \operatorname{Car}(G)$, if $\left(\gamma_{\mathfrak{h}}^{G} \otimes \gamma^{K}\right)(P)$ has a fundamental solution on $H_{0} \times T=A \times B_{0} \times T$, then there exist a constant $C$ and a positive integer $k$ such that

$$
\|P(H, \lambda, \tau)\| \geq \frac{C}{\left(1+|\lambda|^{2}\right)^{k}\left(1+|\tau|^{2}\right)^{k}}
$$

$\forall(\lambda, \tau) \in \mathcal{S}_{d}\left(M_{0}\right) \times \hat{K}$.

Proof. This follows directly from (3) and Theorem 4.1 in [1].

Proposition 4.1 allows us to invert infinitesimal characters of the fundamental series, but we need to do it simultaneously for every $H$-series, $H \in \operatorname{Car}(G)$.

Then we will need the following:

Proposition 4.2. Let $H, J \in \operatorname{Car}(G)$, $H$ fundamental, $P \in \mathcal{Z}\left(\mathcal{U}(\mathfrak{g})^{K}\right)$. If $\left(\gamma_{\mathfrak{h}}^{G} \otimes \gamma^{K}\right)(P)$ has a fundamental solution on $H \times T$, then $\left(\gamma_{\mathfrak{j}}^{G} \otimes \gamma^{K}\right)(P)$ has a fundamental solution on $J_{0} \times T$.

Proof. According to Theorem 4.1 in [1], if $H \in \operatorname{Car}(G)$, then $\left(\gamma_{\mathfrak{h}}^{G} \otimes \gamma^{K}\right)(P)$ has a fundamental solution on $H_{0} \times T$ if and only if

$$
\|P(H, \lambda, \mu)\| \geq \frac{C}{\left(1+|\lambda|^{2}\right)^{k}\left(1+|\mu|^{2}\right)^{k}} \forall(\lambda, \mu) \in \hat{B}_{0} \times \hat{T} .
$$

So we just need to verify (5) for $J_{0} \times T$ provided that it is satisfied for $H \times T$ with $H$ fundamental.

Let $\Gamma$ be a set of strongly orthogonal noncompact imaginary roots such that $\mathfrak{b}^{J}$ is obtained from $\mathfrak{b}$ by Cayley transform $\mathbf{c}_{\Gamma}$. Then $\gamma^{G}=\mathbf{c}_{\Gamma}^{-1} \circ \gamma_{\mathfrak{j}}^{G}$. 
Given $\lambda^{J} \in \mathcal{S}_{d}\left(M_{0}^{J}\right)$, its extension by 0 to $i \mathfrak{b}_{0}^{\prime}$ defines a character in $\hat{B}^{1}$. We write $\lambda$ for this extension, so $\mathbf{c}_{\Gamma}(\lambda)=\lambda^{J}$. Then, if $\nu \in \mathfrak{a}^{\prime}$,

$$
\begin{aligned}
P(H, \lambda, \mu)(\nu) & =\left(\left(\gamma^{G} \otimes \gamma^{K}\right)(P)\right)(\lambda+\nu, \mu) \\
& =\left(\left(\gamma_{j}^{G} \otimes \gamma^{K}\right)(P)\right)\left(\mathbf{c}_{\Gamma}(\lambda+\nu), \mu\right) \\
& =\left(\left(\gamma_{j}^{G} \otimes \gamma^{K}\right)(P)\right)\left(\lambda^{J}+\nu, \mu\right) \\
& =P\left(J, \lambda^{J}, \mu\right)(\nu) .
\end{aligned}
$$

If $P$ has order $m$ in $\mathcal{U}(\mathfrak{g}), P(H, \lambda, \mu)$ and $P\left(J, \lambda^{J}, \mu\right)$ are polynomial functions of order $\leq m$ on $\mathfrak{a}^{\prime}$ and $\left(\mathfrak{a}^{J}\right)^{\prime}$ respectively, and their norms are the norms of the vectors in $\mathbb{C}^{m+1}$ formed with their coefficients, then, by Schwarz inequality,

$$
\|P(H, \lambda, \mu)\| \leq\left\|P\left(J, \lambda^{J}, \mu\right)\right\|
$$

so by hypothesis

$$
\left\|P\left(J, \lambda^{J}, \mu\right)\right\| \geq \frac{\widetilde{C}}{\left(1+\left|\lambda^{J}\right|^{2}\right)^{k}\left(1+|\mu|^{2}\right)^{k}} \quad \forall\left(\lambda^{J}, \mu\right) \in \hat{J}_{0} \times \hat{T} .
$$

\section{Inversion of global characters.}

One important step in building the fundamental solution of $P$ is the construction of distributions $R_{\pi}$ such that $P R_{\pi}=\Theta_{\pi}$ for each representation $\pi$ that appears in the Plancherel formula. In this section we will define these distributions $R_{\pi}$. First we state an analog of Proposition 6.3 in [1] for the $H$-series. We notice that the proof goes exactly the same way.

Proposition 5.1. Let $H \in \operatorname{Car}(G)$. There exist $Z \in \mathcal{Z}(\mathfrak{g})$, a positive constant $C$, an $\varepsilon>0$ and a positive integer $k$ such that $|Z(H, \lambda, \nu+z)| \geq C\left(1+|\lambda|^{2}\right)^{k}\left(1+|\nu|^{2}\right)^{k} \forall \lambda \in \mathcal{S}_{d}\left(M_{0}\right), \nu \in i \mathfrak{a}_{0}^{\prime}, z \in \mathfrak{a}^{\prime},|z|<\varepsilon$.

${ }^{1}$ Although this is a well-known result, we include a sketch of the proof here, since we don't have a reference. Let $T$ be a torus, and $T_{1} \subseteq T$ any subtorus. Then there is a vector space $\mathbb{V}$ and a lattice $\Lambda$ such that $T=\mathbb{V} / \Lambda$. Also $T_{1}=\mathbb{V}_{1} / \Lambda_{1}$, where $\mathbb{V}_{1}$ is a subspace of $\mathbb{V}$ and $\Lambda_{1}=\Lambda \cap \mathbb{V}_{1}$.

It suffices to find another subtorus $T_{2}$ such that $T=T_{1} \times T_{2}$, and for that it suffices to find a sublattice $\Lambda_{2}$ such that $\Lambda=\Lambda_{1} \oplus \Lambda_{2}$.

Let's see first that $\Lambda / \Lambda_{1}$ is torsion free: let $\eta \in \Lambda$ and suppose that $k \eta \in \Lambda_{1}$ for some positive integer $k$. Then $\eta=(1 / k) k \eta \in \mathbb{V}_{1}$ so $\eta \in \Lambda_{1}$.

Now if $\left\{\xi_{1}, \ldots, \xi_{n_{1}}\right\}$ and $\left\{\eta_{1}+\Lambda_{1}, \ldots, \eta_{n_{2}}+\Lambda_{1}\right\}$ are generating sets as free abelian groups of $\Lambda_{1}$ and $\Lambda / \Lambda_{1}$ respectively, then it's easy to see that $\Lambda=\mathbb{Z}\left[\xi_{i}, \eta_{j}\right]$, and we set $\Lambda_{2}=\mathbb{Z}\left[\eta_{j}\right]$.

I wish to thank Prof. Joseph Wolf for giving me this sketch. 
We are now in position to define the distributions $R(H, \lambda, \chi, \nu)$ for $P \in$ $\mathcal{Z}\left(\mathcal{U}(\mathfrak{g})^{K}\right)$ satisfying the hypothesis of Theorem 1.1. Let $\varepsilon>0$ be given by Proposition 5.1, $m$ the order of $P$ and let $\Phi \in C^{\infty}\left(\operatorname{Pol}^{0}(m) \times \mathbb{C}\right)$ be the nonnegative function given by [1, Lemma 6.6].

Given $(\lambda, \chi) \in \mathcal{S}_{d}(M), \tau \in \hat{K}$, and a fixed $\nu \in \mathfrak{a}^{\prime}$, we put

$$
P^{\nu}(H, \lambda, \tau)(z)=P(H, \lambda, \tau)(\nu+z)=P(H, \lambda, \tau, \nu+z) .
$$

Finally, if $d z$ is Lebesgue measure in $\mathfrak{a}^{\prime}, f \in \mathcal{D}(G)$, we define

$$
R(H, \lambda, \chi, \nu)=\sum_{\tau \in \hat{K}} \int_{|z|<\varepsilon} \frac{\Theta^{\tau}(H, \lambda, \chi, \nu+z)}{P^{\nu}(H, \lambda, \tau)(z)} \Phi\left(P^{\nu}(H, \lambda, \tau), z\right) d z .
$$

This definition makes sense because $P^{\nu}(H, \lambda, \tau)(z) \neq 0$ if $\Phi\left(P^{\nu}(H, \lambda, \tau), z\right) \neq$ 0 ([1, Lemma 6.6 (iv) $])$.

Proposition 5.2. The map defined by (7) is a finite order distribution for all $(\lambda, \chi) \in \mathcal{S}_{d}(M), \nu \in \mathfrak{a}^{\prime}$. This map has also the following properties:

(i) $\operatorname{PR}(H, \lambda, \chi, \nu)=\Theta(H, \lambda, \chi, \nu)$.

(ii) For every positive integer $k$ and $f \in \mathcal{D}(G)$, exist a constant $C>0$ which only depends on the support of $f$ and a differential operator $D_{k} \in \mathcal{Z}\left(\mathcal{U}(\mathfrak{g})^{K}\right)$ such that $\forall(\lambda, \chi) \in \mathcal{S}_{d}(M), \nu \in i \mathfrak{a}_{0}^{\prime}$

$$
|R(H, \lambda, \chi, \nu)(f)| \leq \frac{C}{\left(1+|\nu|^{2}\right)^{k}\left(1+|\lambda|^{2}\right)^{k}}\left\|D_{k} f\right\|_{L^{2}(G)} .
$$

Proof. Although the argument is the same as [1, Proposition 6.8] for $M A N$ minimal parabolic, we will sketch it here in order to verify that every step of the argument still applies for $M A N$ any cuspidal parabolic.

(i) is clear, combining (4) with [1, Lemma 6.6 (iii)] and the fact that $P(H, \lambda, \tau, \nu+z)$ is holomorphic in $\mathrm{z}$.

Let's see that (7) defines a distribution: According to [1, Lemma 6.6 (iv)] and [1, Lemma 6.7] together with Proposition 4.2, it holds, for all $|z|<\varepsilon$ and some $\widetilde{k}$,

$$
\left|\frac{\Phi\left(P^{\nu}(H, \lambda, \tau), z\right)}{P^{\nu}(H, \lambda, \tau)(z)}\right| \leq C_{1}\left(1+|\nu|^{2}\right)^{m}\left(1+|\lambda|^{2}\right)^{\widetilde{k}}\left(1+|\tau|^{2}\right)^{\widetilde{k}} .
$$

On the other hand, if $Z \in \mathcal{Z}(\mathfrak{g})$ and $\Omega \in \mathcal{Z}(\mathfrak{k})$ are given by Proposition 5.1 and [1, Lemma 6.1] respectively, and if $s_{1}$ and $s_{2}$ are positive integers, we have, for some positive integer $\bar{k}$,

$$
\left|\Theta^{\tau}(H, \lambda, \chi, \nu+z)(f)\right| \leq \frac{C_{2}\left|\Theta(H, \lambda, \chi, \nu+z)\left(\left(Z^{t}\right)^{s_{1}}\left(\Omega^{t}\right)^{s_{2}} f\right)\right|}{\left(\left(1+|\lambda|^{2}\right)\left(1+|\nu|^{2}\right)\right)^{s_{1}-\bar{k}}\left(1+|\tau|^{2}\right)^{s_{2}}} .
$$

Let $\widetilde{K} \subseteq G$ be a compact subset. Note that for $H$-series representations the multiplicity $n_{\tau}$ of any $K$-type satisfies $n_{\tau} \leq \operatorname{dim} \tau([\mathbf{8}$, p. 207]); so 
by $[\mathbf{1}$, Lemma 6.5$]$ exist $\widetilde{\Omega} \in \mathcal{Z}(\mathfrak{k})$ and a constant $C_{3}$ independent of $\lambda \in$ $\mathcal{S}_{d}\left(M_{0}\right), \nu, z \in \mathfrak{a}^{\prime}$ such that

$$
|\Theta(H, \lambda, \chi, \nu+z)(f)| \leq C_{3}\left(\int_{G}|\widetilde{\Omega} f(g)|^{2}\|\pi(H, \lambda, \chi, \nu+z)(g)\|^{2} d g\right)^{1 / 2} .
$$

On the other hand, given $\varphi$ in the space where $\pi(H, \lambda, \chi, \nu+z)$ acts, if $a(g)$ is the $A$-component of $g$ in the $K M A N$ decomposition, then (cf. [8, p. 169]),

$$
(\pi(H, \lambda, \chi, \nu+z)(g) \varphi)(k)=e^{-z \log a\left(g^{-1} k\right)}(\pi(H, \lambda, \chi, \nu)(g) \varphi)(k),
$$

and taking $A=\sup _{g \in \widetilde{K}, k \in K,|z|<\varepsilon}\left|e^{-z \log a\left(g^{-1} k\right)}\right|$ and $B_{\lambda, \nu}=\sup _{g \in \widetilde{K}}\|\pi(H, \lambda, \chi, \nu)(g)\|$, then $\|\pi(H, \lambda, \chi, \nu+z)(g)\| \leq A B_{\lambda, \nu}$ uniformly on $\widetilde{K}$, so for all $f$ supported in $\widetilde{K}$,

$$
\left|\Theta^{\tau}(H, \lambda, \chi, \nu+z)(f)\right| \leq A B_{\lambda, \nu}\|\widetilde{\Omega} f\|_{L^{2}(G)} .
$$

Now combining (8), (9) and (10) we obtain, for all $f$ supported in $\widetilde{K}$,

$$
\begin{aligned}
& |R(H, \lambda, \chi, \nu)(f)| \\
& \leq\left(\sum_{\tau \in \hat{K}} \frac{1}{\left(1+|\tau|^{2}\right)^{s_{2}-\widetilde{k}}}\right) \frac{C_{4} B_{\lambda, \nu}\left\|\widetilde{\Omega}\left(Z^{t}\right)^{s_{1}}\left(\Omega^{t}\right)^{s_{2}} f\right\|_{L^{2}(G)}}{\left(1+|\nu|^{2}\right)^{s_{1}-m-\bar{k}}\left(1+|\sigma|^{2}\right)^{s_{1}-\widetilde{k}-\bar{k}}}
\end{aligned}
$$

and $\sum_{\tau \in \hat{K}} \frac{1}{\left(1+|\tau|^{2}\right)^{s_{2}-\widetilde{k}}}$ is finite if we choose $s_{2}>\widetilde{k}+1 / 2 \operatorname{dim} K$. Hence $R_{\sigma, \nu}$ is a finite order distribution.

To see (ii), just observe that $B_{\lambda, \nu}=1$ if $\nu \in i \mathfrak{a}_{0}^{\prime}$, so given $k$ if we take $D_{k}=\widetilde{\Omega}\left(Z^{t}\right)^{s_{1}}\left(\Omega^{t}\right)^{s_{2}}$ with the $s_{2}$ chosen above and $s_{1} \geq k+\bar{k}+\max (\widetilde{k}, m)$, (11) becomes

$$
|R(H, \lambda, \chi, \nu)(f)| \leq \frac{C}{\left(1+|\nu|^{2}\right)^{k}\left(1+|\lambda|^{2}\right)^{k}}\left\|D_{k} f\right\|_{L^{2}(G)}
$$

with $C$ depending only $\widetilde{K}$.

\section{Demonstration of Theorem 1.1.}

Now we are ready to complete the proof of Theorem 1.1 with the explicit construction of the fundamental solution of $P$.

Proposition 6.1. Let $P \in \mathcal{Z}\left(\mathcal{U}(\mathfrak{g})^{K}\right)$. Suppose that for each $H \in \operatorname{Car}(G)$ there exist a constant $C$ and a positive integer $k$ such that

$$
\|P(H, \lambda, \tau)\| \geq \frac{C}{\left(1+|\lambda|^{2}\right)^{k}\left(1+|\tau|^{2}\right)^{k}} \quad \forall(\lambda, \tau) \in \mathcal{S}_{d}\left(M_{0}\right) \times \hat{K} .
$$


If $R(H, \lambda, \chi, \nu)$ are the distributions defined by (7), then the map $R$ defined by

$$
R=\sum_{H \in \operatorname{Car}(G)}\left(\sum_{(\lambda, \chi) \in \mathcal{S}_{d}(M)} \int_{\nu \in i \mathfrak{a}_{0}^{\prime}} R(H, \lambda, \chi, \nu) m(H, \lambda, \chi, \nu) d \nu\right)
$$

is a finite order distribution which is a fundamental solution of $P$.

Remark. If $P \in \mathcal{Z}\left(\mathcal{U}(\mathfrak{g})^{K}\right)$ is such that $\left(\gamma^{G} \otimes \gamma^{K}\right)(P)$ has a fundamental solution in $H \times T$, Propositions 4.1 and 4.2 imply (12) for all $H \in \operatorname{Car}(G)$, so Theorem 1.1 is a direct consequence of Proposition 6.1.

Proof. Equality $P R=\delta$ is clear by Plancherel formula (Theorem 2.1) and because $P R(H, \lambda, \chi, \nu)=\Theta(H, \lambda, \chi, \nu)$; it only remains to prove that $R$ is a finite order distribution. For that we will prove that each of the following are finite order distributions:

$$
R_{H}=\sum_{(\lambda, \chi) \in \mathcal{S}_{d}(M)} \int_{\nu \in i \mathfrak{a}_{0}^{\prime}} R(H, \lambda, \chi, \nu) m(H, \lambda, \chi, \nu) d \nu .
$$

Let $\widetilde{K}$ be a compact subset and $f \in \mathcal{D}_{\widetilde{K}}(G)$; for each positive integer $k$ let $D_{k} \in \mathcal{Z}\left(\mathcal{U}(\mathfrak{g})^{K}\right)$ be given by Proposition 5.2 (ii); then, using Theorem 2.1 (ii),

$\left|R_{H}(f)\right| \leq C_{1} C_{2}\left(\sum_{\lambda \in \mathcal{S}_{d}\left(M_{0}\right)} \frac{1}{\left(1+|\lambda|^{2}\right)^{k-l_{2}}}\right)\left(\int_{\nu \in i \mathfrak{a}_{0}^{\prime}} \frac{1}{\left(1+|\nu|^{2}\right)^{k-l_{1}}}\right)\left\|D_{k} f\right\|_{L^{2}(G)}$.

Choosing $k$ large enough so that the sum and the integral are finite, we obtain an operator $D \in \mathcal{Z}\left(\mathcal{U}(\mathfrak{g})^{K}\right)$ and a constant $C$ depending only on $\widetilde{K}$ such that

$$
\left|R_{H}(f)\right| \leq C\|D f\|_{L^{2}(G)},
$$

and this proves that $R_{H}$ is a distribution of finite order less or equal that the order of $D$.

\section{Final remarks.}

7.1. P-convexity of $G$. Suppose that $P \in \mathcal{Z}\left(\mathcal{U}(\mathfrak{g})^{K}\right)$ satisfies the conditions of Proposition 6.1. The existence of fundamental solution of $P$ implies that the differential equation $P u=f$ has a solution $u \in C^{\infty}(G)$ for all $f \in \mathcal{D}(G)$. Now, in order to guarantee the solvability of $P u=f$ when $f \in C^{\infty}(G)$, it is necessary to analyze the $P$-convexity of $G$.

We have done this already in $[\mathbf{1}, \S 8]$. We just have to notice that that argument applies for general linear semisimple groups, since Johnson's injectivity criterion (Theorems 5.1 and 5.2 in [7]) holds for these groups. 
7.2. Casimir operator. Let $\Omega \in \mathcal{Z}(\mathfrak{g})$ be the Casimir operator of $G$. As it was remarked in [2], Corollary 1.2 provides a fundamental solution of $\Omega$ only if $G$ doesn't have a compact Cartan subgroup. For the sake of completeness, we recall the argument.

If $\mathfrak{h}$ is a Cartan subalgebra of $\mathfrak{g}$, and if $\lambda \in \mathfrak{h}^{\prime}$, then $\chi_{\lambda}(\Omega)=B(\lambda, \lambda)-$ $B(\rho, \rho)$; in particular, if $H \in \operatorname{Car}(G), \mathfrak{h}=\mathfrak{b} \oplus \mathfrak{a}, \lambda \in \hat{B}_{0}, \nu \in i \mathfrak{a}_{0}^{\prime}$, then

$$
\Omega(H, \lambda, \nu)=\chi_{\lambda+\nu}(\Omega)=|\lambda|^{2}-|\nu|^{2}-|\rho|^{2},
$$

so if $\mathfrak{a} \neq 0,\|\Omega(H, \lambda)\| \geq 1$ for all $\lambda \in \hat{B}_{0}$, and $\gamma_{\mathfrak{h}}^{G}(\Omega)$ has a fundamental solution on $H_{0}$. Now if $G$ has a compact Cartan subgroup $B$

$$
\Omega(B, \lambda)=|\lambda|^{2}-|\rho|^{2} .
$$

Since $G^{\mathbb{C}}$ is simply connected, $\lambda=\rho$ is a character of $B$, and consequently we cannot apply Corollary 1.2 in this case.

7.3. Operators of $\mathcal{Z}(\mathfrak{k})$. Let's consider $P \in \mathcal{Z}(\mathfrak{k}) \subset \mathcal{Z}\left(\mathcal{U}(\mathfrak{g})^{K}\right)$. Then $P$ can be seen as a differential operator acting on both $G$ and $K$. Now, according to [4, Theorem I], $P$ has a fundamental solution on $K$ if and only if there exists $C>0, k \in \mathbb{N}$ such that

$$
|P(\tau)| \geq \frac{C}{\left(1+|\tau|^{2}\right)^{k}} \quad \forall \tau \in \hat{K} .
$$

We are in position to prove the following:

Proposition 7.1. If $P \in \mathcal{Z}(\mathfrak{k}) \subset \mathcal{Z}\left(\mathcal{U}(\mathfrak{g})^{K}\right)$ has a fundamental solution on $K$, then it has one in $G$.

Proof. All we have to do at this point is notice that inequality (16) is nothing else than (12) for $P \in \mathcal{Z}(\mathfrak{k})$, so we can apply Proposition 6.1 directly.

Let's finish analyzing the case of $\Omega_{K} \in \mathcal{Z}(\mathfrak{k})$ the Casimir of $K$. In this case we have (see $[\mathbf{1}, \S 11]$ )

$$
\gamma_{\mathfrak{a}} \otimes \chi_{\tau}\left(\Omega_{K}\right)=\chi_{\tau}\left(\Omega_{K}\right)=\left|\lambda_{\tau}\right|^{2}-\left|\rho_{K}\right|^{2} .
$$

So if we choose $\tau$ such that $\lambda_{\tau}=\rho_{K}$, by [1, Prop. 11.1], $\Omega_{K}$ doesn't have a parametrix.

However, if we take $\Omega_{K}+C$ such that $C>\left|\rho_{K}\right|^{2}$, then

$$
\left(\Omega_{K}+C\right)(H, \lambda, \mu, \nu)=\chi_{\mu}\left(\Omega_{K}+C\right) \geq \text { Constant }
$$

and Theorem 1.1 applies, therefore $\Omega_{K}+C$ does have a fundamental solution. 
Acknowledgments. I wish to thank Prof. Joseph Wolf, who is my advisor as a postdoctoral fellow in Berkeley, for all his support during this period; and particularly for his help with many topics of this paper.

I also want to thank my friend Dr. Leandro Cagliero for his unconditional will to spend his time to read, discuss, and help me with this work.

Finally, I'd like to thank the Mathematics Department of the University of California at Berkeley.

\section{References}

[1] G. Ames, Fundamental solutions of invariant differential operators on a semisimple Lie group, Pacific J. Math., 196(1) (2000), 17-44, MR 2002h:22013.

[2] A. Benabdallah and F. Rouvière, Solvability of bi-invariant operators on a semisimple Lie group, C.R. Acad. Sc. Paris, Serie I, 298(17) (1984), 405-408, MR 85j:22017, Zbl 0564.22013.

[3] A. Bouaziz and N. Kamoun, Équations différentielles sur les groupes et algèbres de Lie réductifs, Ann. Inst. Fourier, 50(6) (2000), 1799-1857, MR 2002i:22013, Zbl 0970.22009.

[4] A. Cerezo and F. Rouvière, Solution élémentaire d'un opérateur invariant à gauche sur un groupe de Lie réel compact, Ann. Scient. Éc. Norm. Sup., 2 (1969), 561-581, MR 42 \#6869, Zbl 0191.43801.

[5] R. Herb and J.A. Wolf, The Plancherel theorem for semisimple Lie groups, Compositio Mathematica, 57 (1986), 271-355, MR 87h:22020, Zbl 0587.22005.

[6] _ Rapidly decreasing functions on general semisimple groups, Compositio Mathematica, 58 (1986), 73-110, MR 87f:22014, Zbl 0587.22006.

[7] K. Johnson, Differential equations and an analog to Paley-Wiener theorem for linear semisimple Lie groups, Nagoya Math. Journal, 64 (1976), 17-29, MR 58 \#1025, Zbl 0329.43008.

[8] A. Knapp, Representation Theory of Semisimple Groups, Princeton University Press, 1986, MR 87j:22022, Zbl 0604.22001.

[9] F. Knop, Harish-Chandra homomorphisms for reductive group actions, Ann. of Math., 140 (1994), 253-288, MR 95h:14045, Zbl 0828.22017.

Received March 1, 2001 and revised May 30, 2001. This work was supported by CONICET.

Department of Mathematics

UNIVERSITY OF CALIFORNIA

BERKELEY, CA 94720

E-mail address: ames@math.berkeley.edu 